\title{
CARACTERIzAción PAlinológica de Mieles de la Zona I: Prepuna. (JuJuY, ARgENTINA)
}

\author{
ANA C. SÁNCHEZ y y LILIANA C. LUPO 1
}

\begin{abstract}
Resumen: Se realizó el análisis palinológico de once muestras de miel pertenecientes a la Zona I, Prepuna en la provincia de Jujuy. Se determinaron 38 tipos polínicos, destacándose aquellos correspondientes a especies nativas con $39 \%$. Se identificaron como principales recursos nectaríferos Prosopis ferox, Tipo Papilionoideae, Arquita trichocarpa, Brassicaceae y Schinus areira y como asociación indicadora del origen geográfico: Prosopis ferox, Schinus areira, Brassicaceae, Caesalpinia trichocarpa, Papilionoideae, Plantago sp., Baccharis sp., Mutisieae, Poaceae, Asteraceae, Solanaceae y Verbenaceae.
\end{abstract}

Palabras clave: Melisopalinología, Polen, Quebrada de Huahuaca, Apis mellifera.

\begin{abstract}
Summary: Palynological characterization of honey from Zone I: Prepuna (Jujuy, Argentine). We performed palynological analysis of the eleven honey samples belonging to Zone I Prepuna in Jujuy province. Thirty eight pollen types were identified, highlighting those correspond to native species with $39 \%$. Prosopis ferox, Papilionoideae type, Arquita trichocarpa, Brassicaceae y Schinus areira were identified as main nectar resources and as association of indicator of geographical origin: Prosopis ferox, Schinus areira, Brassicaceae, Caesalpinia trichocarpa, Papilionoideae type, Plantago sp., Baccharis sp., Mutisieae, Poaceae, Asteraceae, Solanaceae y Verbenaceae.
\end{abstract}

Key words: Melissopalynology, Pollen, Quebrada de Humahuaca, Apis mellifera.

\section{INTRODUCCIÓN}

La caracterización botánica y geográfica de mieles es una herramienta de valor en la comercialización, ya que es conocido que el análisis microscópico de las mieles, permite identificar el recurso nectarífero utilizado por Apis mellifera y las áreas donde fueron producidas (Ricciardelli D'Albore, 1997). En la provincia de Jujuy la apicultura es una actividad complementaria, que se lleva a cabo en forma artesanal y cumple un rol social y económico. En este contexto los volúmenes producidos y comercializados representan un porcentaje bajo a nivel nacional (MAGYP, 2015), por ello la caracterización ofrece la oportunidad de

\footnotetext{
1 Laboratorio de Palinología. Instituto de Ecoregiones Andinas (INECOA), Universidad Nacional de Jujuy CONICET, Facultad de Ciencias Agrarias, (Alberdi $47 \mathrm{~B}^{\circ}$ Los Naranjos, 4.600), S. S. de Jujuy, Argentina. E-mail lab.palinologia@fca.unju.edu.ar
}

incorporar valor a este importante producto de la colmena.

Con esta finalidad, se realizaron estudios melisopalinológicos de mieles, procedentes de distintos ambientes, los que constituyeron los primeros aportes a este conocimiento (Sánchez \& Lupo, 2011; Sánchez, 2013). Éstas investigaciones permitieron establecer, desde el punto de vista palinológico, tres zonas apícolas en la provincia: Zona I, Prepuna; Zona II: Yungas y Zona III: Transición Yungas-Chaco (Fig. 1). Cabe destacar que en el área mencionada se han llevado a cabo estudios sobre la flora melífera (Sánchez \& Vignale, 2009), donde se ha observado la preferencia de $A$. mellifera por la utilización de especies nativas. En esta contribución se presentan los resultados obtenidos para la Zona I Prepuna, correspondiente a la Provincia Fitogeográfica de la Prepuna, conocida como Quebrada de Humahuaca, la que fue declarada Patrimonio Cultural y Natural de la Humanidad en el año 2003. 


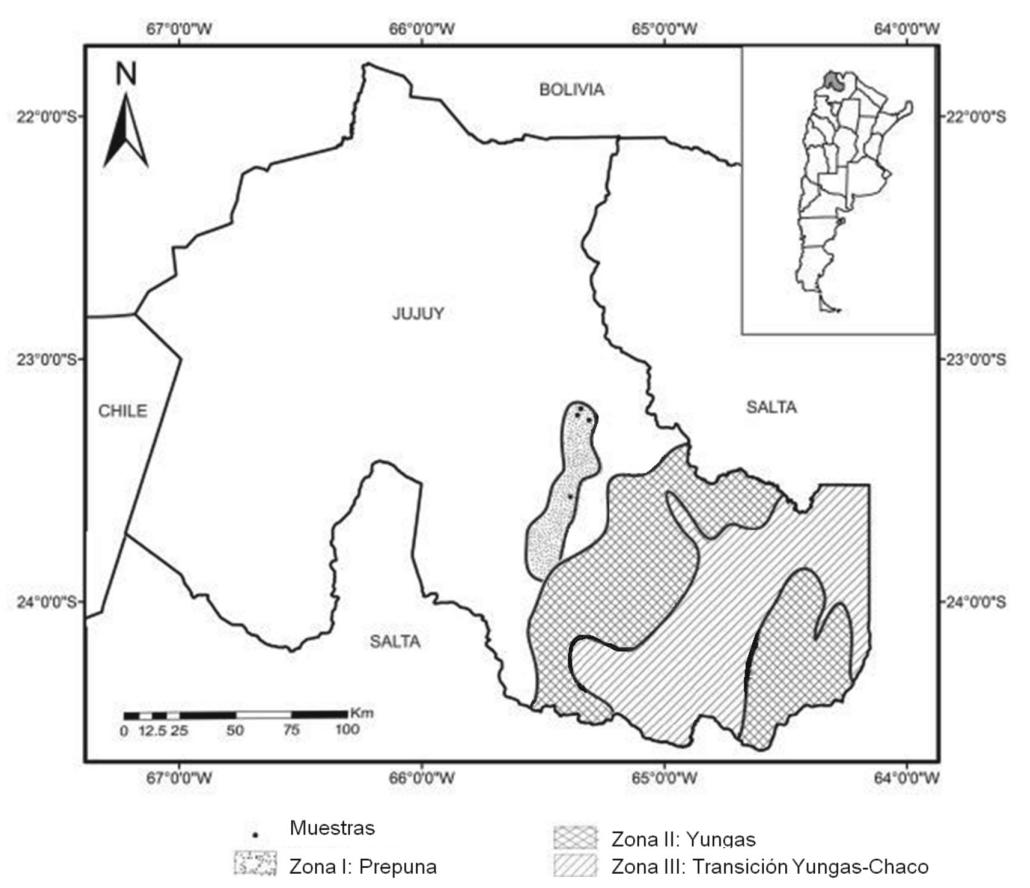

Fig. 1. Zonas apícolas de Jujuy.

\section{Materiales y Métodos}

La zona de muestreo se encuentra comprendida en la provincia fitogeográfica de la Prepuna (Cabrera, 1976), la que se extiende por las laderas y quebradas secas entre 2.000 y 3.400 msnm (Fig. 1), caracterizándose por la estepa arbustiva de Senna crassiramea (Benth.) H.S. Irwin \& Barneby (sumalahua), Arquita trichocarpa (Griseb.) E. Gagnon, G.P. Lewis \& C.E. Hughes. y Cercidium andicola Griseb., entre otras, además de bosquesillos de cardones (Trichocereus atacamensis (Phil.) Backeb. y Trichocereus tarijensis (Vaupel) Werderm.; comunidades edáficas de churqui (Prosopis ferox Griseb.), en quebradas secas; matorrales de molle (Schinus areira L.) y Baccharis salicifolia (Ruiz \& Pav.) Pers. en orillas de ríos y cojines de Bromeliaceae en laderas rocosas.

Se analizaron 11 muestras de miel cosechadas en el período comprendido entre los años 2004 y 2013, procedentes de las localidades de Humahuaca, Ocumazo y San Roque en el Dto. Humahuaca y de Tilcara, Dto. Tilcara (Fig. 1). Las muestras se extrajeron por centrifugado, procurando que sean representativas de cada apiario mediante la homogeneización. Los análisis melisopalinológicos se realizaron siguiendo la metodología propuesta por Louveaux et al. (1978). Las observaciones se efectuaron con Microscopio Óptico Zeiss ICS KF2 y las fotografías se tomaron con microscopio Leica DM500 con cámara incorporada Leica ICC50. La determinación de los tipos polínicos se efectuó mediante la comparación con la Palinoteca de Referencia del Laboratorio Palinología, Facultad de Ciencias Agrarias, Universidad Nacional de Jujuy (PALJUA). Se consultaron también los siguientes atlas: Markgraf \& D' Antoni (1978) y Pire et al. (1998, 2002, 2006). Cuando fue posible se identificaron los tipos polínicos a nivel de especie a excepción de aquellos casos en que se llegó a nivel genérico, de tribu, subfamilia o familia.

Se definieron las siguientes clases de frecuencias: polen dominante " $\mathrm{D}$ " (>45\%); polen secundario "S" (16-45\%), polen de menor importancia "M" (3- 15\%) y polen en traza "T" $(<3 \%)$ (Louveaux et al., 1978), considerándose mieles monoflorales cuando presentaron un tipo polínico dominante. La frecuencia de ocurrencia (Feller-Demalsy et 
al., 1987) se estableció como el porcentaje en que aparece un tipo polínico en el total de las muestras: muy frecuente "MF" (>50\%); frecuente "F" (20$50 \%)$; poco frecuente "PF" $(10-20 \%)$ y raro "R" $(<10 \%)$.

Para el origen geográfico se clasificaron los tipos polínicos en: nativos, exóticos y origen indeterminado. Con esa información se confeccionó una base de datos para la elaboración del diagrama polínico utilizando el programa TILIA 1.7.14 (Grimm, 1992), considerándose como indicadores a los tipos polínicos muy frecuentes.

\section{Resultados}

Se determinaron 38 tipos polínicos pertenecientes a 23 familias botánicas, destacándose las Asteraceae con siete tipos, Fabaceae con cinco, Solanaceae con tres, Amaranthaceae con dos y el resto de las familias con un representante cada una (Tabla 1, Fig. 2). La riqueza de tipos polínicos por muestra varió entre ocho y 21 , siendo el promedio 15 . Según la clase de frecuencia se han registrado diez muestras multiflorales y una unifloral de Prosopis ferox. Según la frecuencia de ocurrencia en el conjunto de las muestras, los tipos polínicos clasificados como muy frecuentes son Prosopis ferox (100\%), Schinus areira (100\%), Brassicaceae (91\%), Arquita trichocarpa (91\%), tipo Papilionoideae (91\%), Plantago sp. (91\%), Baccharis sp. (82\%), Mutisieae (82\%), acompañados de Poaceae (64\%), Asteraceae (55\%), Solanaceae (55\%) y Verbenaceae (55\%), Se observa que los elementos de origen nativo (39\%) son más importantes que los introducidos (29\%) y los de origen indeterminado (32\%) (Figs. 2 y 3 ).

\section{Discusıón}

De los 38 tipos polínicos hallados en las mieles, se observa que 12 de ellos fueron registrados en el estudio de flora apícola (Sánchez \& Vignale, 2009). Se destacan Prosopis ferox y Senecio tilcarensis como intensamente usados, siempre visitados, aunque solamente la primera se observa en la miel como muy frecuente alcanzando porcentaje de polen secundario y dominante, mientras que la segunda solo se encuentra en trazas en una muestra de miel procedente de la localidad de
Tilcara. Schinus areira, Baccharis sp., Cichorium intybus, Taraxacum officinale y Nicotiana glauca se los mencionan como muy usadas, siempre visitados, observándose en las mieles el aporte de S. areira en el $100 \%$ de las muestras, con clase de frecuencia que varía entre polen secundario y trazas y el de Baccharis sp. en el $82 \%$ de las muestras aunque con menor clase de frecuencia. Arquita trichocarpa se la clasificó como poco usada, visitada esporádicamente por abejas, pero el aporte de esta se aprecia en la miel, con frecuencia de ocurrencia del $91 \%$ y clase de frecuencia que varía entre secundario y trazas. El aporte de las demás especies no se observa como importante ya que se registraron como poco frecuentes y en trazas en las muestras. Las diferencias encontradas entre las observaciones de la actividad de las abejas en las flores y el aporte de polen a las mieles, podría estar relacionado a las variaciones en la abundancia de estos recursos en las proximidades de los apiarios y a los cambios de las condiciones climáticas que afectan la floración.

Las familias más importantes por el número de representantes encontrados en el total de las muestras son Asteraceae con siete tipos, Fabaceae con cinco, al igual que sucede en otros ambientes de la provincia (Sánchez, 2013) y del país (Tellería, 1992; 1995; 1996a; 1996b; Basilio \& Romero, 1996; Forcone y Tellería, 1998; Andrada \& Tellería 2002; Fagundez \& Caccavari, 2003, 2006; Lusardi et al. 2005; Cabrera, 2006; Naab \& Tamame, 2007; Forcone, 2008). Las Fabaceae y Asteraceae también son importantes en las mieles de México (Villanueva-Gutiérrez et al., 2009; Ramírez-Arriaga et al., 2014), mientras que en las Caatingas, Brasil, las Fabaceae se registran con mayor representación seguida por Euphorbiaceae, Rubiaceae ubicándose Asteraceae en cuarto lugar (Oliveira \& Ribeiro dos Santos, 2014). En Europa, Persano Odo et al. (2007) menciona estas dos familias como las de mayor número de representantes en mieles de Italia y en el noroeste de España se las cita junto con las Ericaceae (Seijo et al., 1992, 1994,1995; Iglesias et al., 1993).

Como recurso nectarífero utilizado por las abejas se destacan las Fabaceae: Prosopis ferox, presente en el $100 \%$ de las muestras encontrándose en una de ellas como polen dominante y en tres como secundario; Papilionoideae y Arquita trichocarpa en el $91 \%$ de las muestras, alcanzando valores 
Bol. Soc. Argent. Bot. 51 (3) 2016

Tabla 1. Tipos polínicos identificados en las mieles de la Zona I: Prepuna. Clases de Frecuencias: D (Dominante), S (Secundario), M (Menor Importancia) y T (Trazas). FO: Frecuencia de Ocurrencia en el total de las muestras. (P) Especies poliniferas.

\begin{tabular}{|c|c|c|c|c|c|c|c|c|c|c|c|c|c|}
\hline \multirow{2}{*}{ Familia } & \multirow{2}{*}{ Tipo polínico } & \multicolumn{11}{|c|}{ Muestras } & \multirow{2}{*}{$\begin{array}{l}\text { FO } \\
\%\end{array}$} \\
\hline & & 79 & 218 & 220 & 238 & 241 & 356 & 443 & 477 & 491 & 492 & 524 & \\
\hline Acanthaceae & Acanthaceae & & $\mathrm{T}$ & & & & & & & & & & 9 \\
\hline \multirow[t]{2}{*}{ Amaranthaceae } & Amaranthaceae $(P)$ & & & & & & & $\mathrm{T}$ & & & & & 9 \\
\hline & Gomphrena sp. (P) & & & & & & $\mathrm{T}$ & & & & & & 9 \\
\hline Anacardiaceae & Schinus areira L. & $\mathrm{T}$ & M & $S$ & $\mathrm{~S}$ & M & M & $\mathrm{T}$ & $\mathrm{T}$ & $\mathrm{T}$ & M & $S$ & 100 \\
\hline Apiaceae & Apiaceae & M & & & $\mathrm{T}$ & & $\mathrm{T}$ & $\mathrm{T}$ & & & & & 36 \\
\hline \multirow[t]{7}{*}{ Asteraceae } & Asteraceae & $\mathrm{T}$ & & $\mathrm{T}$ & & & M & $\mathrm{T}$ & & M & M & & 55 \\
\hline & Baccharis sp. & $\mathrm{T}$ & $\mathrm{T}$ & M & $\mathrm{T}$ & M & $\mathrm{T}$ & $\mathrm{T}$ & $\mathrm{T}$ & M & & & 82 \\
\hline & Cichorium intybus L. & & & $\mathrm{T}$ & & & & & & & & & 9 \\
\hline & Mutisieae & $\mathrm{T}$ & $\mathrm{T}$ & $\mathrm{T}$ & $\mathrm{T}$ & S & $\mathrm{T}$ & $\mathrm{T}$ & & $\mathrm{T}$ & $\mathrm{T}$ & & 82 \\
\hline & Senecio tilcarensis Cabrera & $\mathrm{T}$ & & & & & & & & & & & 9 \\
\hline & Sonchus sp. & & & & & & & & & $\mathrm{T}$ & $\mathrm{T}$ & & 18 \\
\hline & Taraxacum officinale F.H. Wigg. & & & & & & $\mathrm{T}$ & & & & & & 9 \\
\hline Brassicaceae & Brassicaceae & S & M & M & M & M & S & M & $\mathrm{T}$ & S & S & & 91 \\
\hline Boraginaceae & Boraginaceae & & & & & & $\mathrm{T}$ & & $\mathrm{T}$ & & & & 18 \\
\hline Buddlejaceae & Buddleja sp. & $\mathrm{T}$ & M & M & M & & $\mathrm{T}$ & & & & & & 45 \\
\hline Cactaceae & Trichocereus sp. & $\mathrm{T}$ & $\mathrm{T}$ & & & & & $\mathrm{T}$ & & $\mathrm{T}$ & & $\mathrm{T}$ & 45 \\
\hline \multirow[t]{5}{*}{ Fabaceae } & Acacia sp. & $\mathrm{T}$ & & $\mathrm{T}$ & & $\mathrm{T}$ & & & & & & & 27 \\
\hline & $\begin{array}{l}\text { Arquita trichocarpa (Griseb.) E. } \\
\text { Gagnon, G.P. Lewis \& C.E. Hughes }\end{array}$ & $\mathrm{T}$ & & M & M & $\mathrm{S}$ & $\mathrm{T}$ & S & $\mathrm{T}$ & M & M & M & 91 \\
\hline & Cercidium andicola Griseb. & $\mathrm{T}$ & & $\mathrm{T}$ & & & & & $\mathrm{T}$ & & & & 27 \\
\hline & Papilionoideae & S & $\mathrm{T}$ & S & $\mathrm{S}$ & M & S & S & & M & M & M & 91 \\
\hline & Prosopis ferox Griseb. & M & $S$ & M & M & M & $\mathrm{T}$ & M & $\mathrm{D}$ & S & S & M & 100 \\
\hline Lamiaceae & Lamiaceae & & & & & $\mathrm{T}$ & & & & $\mathrm{T}$ & & & 18 \\
\hline Malvaceae & Malvaceae & & & $\mathrm{T}$ & & & $\mathrm{T}$ & & & & & & 18 \\
\hline Myrtaceae & Eucalyptus sp. & M & & & $\mathrm{T}$ & $S$ & $\mathrm{~T}$ & $\mathrm{~T}$ & $\mathrm{~T}$ & & & & 55 \\
\hline Oleaceae & Ligustrum lucidum W.T. Aiton & & $\mathrm{T}$ & & & & & & & & & & 9 \\
\hline Plantaginaceae & Plantago sp. (P) & & M & $\mathrm{T}$ & $\mathrm{T}$ & $\mathrm{T}$ & M & M & $\mathrm{T}$ & $\mathrm{M}$ & M & S & 91 \\
\hline Poaceae & Poaceae & $\mathrm{T}$ & & $\mathrm{T}$ & & & M & $\mathrm{T}$ & & $\mathrm{T}$ & $\mathrm{T}$ & M & 64 \\
\hline Rhamnaceae & Rhamnaceae & M & & & & & & & & & & & 9 \\
\hline Rosaceae & Rosaceae & & $\mathrm{T}$ & & & & & & & & & & 9 \\
\hline Salicaceae & Salix humboldtiana Willd. & & $\mathrm{T}$ & & & & & & & & & & 9 \\
\hline Scrophulariaceae & Veronica sp. & $\mathrm{T}$ & & & & & & & & & & & 9 \\
\hline \multirow[t]{3}{*}{ Solanaceae } & Solanaceae & M & M & $\mathrm{T}$ & & & $\mathrm{T}$ & $\mathrm{T}$ & $\mathrm{T}$ & & & & 55 \\
\hline & Lycium sp. & & & & & & $\mathrm{T}$ & & $\mathrm{T}$ & $\mathrm{T}$ & $\mathrm{T}$ & & 36 \\
\hline & Nicotiana glauca Graham & & & M & & & & & & & & & 9 \\
\hline $\begin{array}{l}\text { Urticaceae- } \\
\text { Moraceae }\end{array}$ & Urticaceae-Moraceae (P) & & & & & $\mathrm{T}$ & & & & & & & 9 \\
\hline \multirow[t]{3}{*}{ Verbenaceae } & Verbenaceae & & & & $\mathrm{T}$ & & $\mathrm{T}$ & M & & $\mathrm{T}$ & $\mathrm{T}$ & $\mathrm{T}$ & 55 \\
\hline & Monocotiledonea & & M & & & $\mathrm{T}$ & & & & & & & 18 \\
\hline & Indeterminado & & $\mathrm{T}$ & $\mathrm{T}$ & & $\mathrm{T}$ & $\mathrm{T}$ & & & & & & 36 \\
\hline Riqueza de espeies & & 15 & 12 & 11 & 12 & 8 & 16 & 18 & 21 & 14 & 19 & 16 & \\
\hline
\end{tabular}


A. C. Sánchez y L. C. Lupo - Caracterización palinológica de mieles

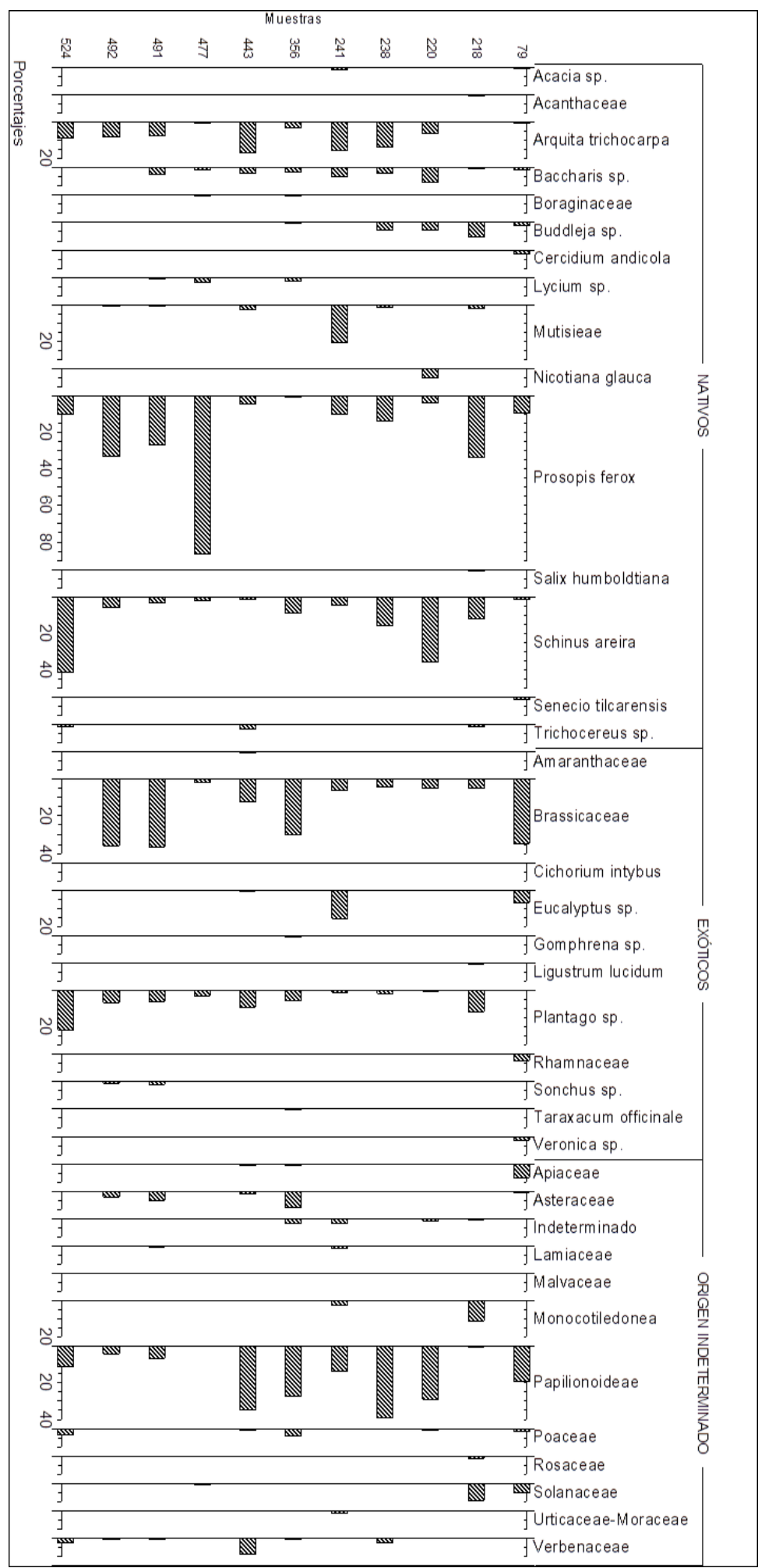

Fig. 2. Espectro polínico de las mieles de la Zona I: Prepuna. 

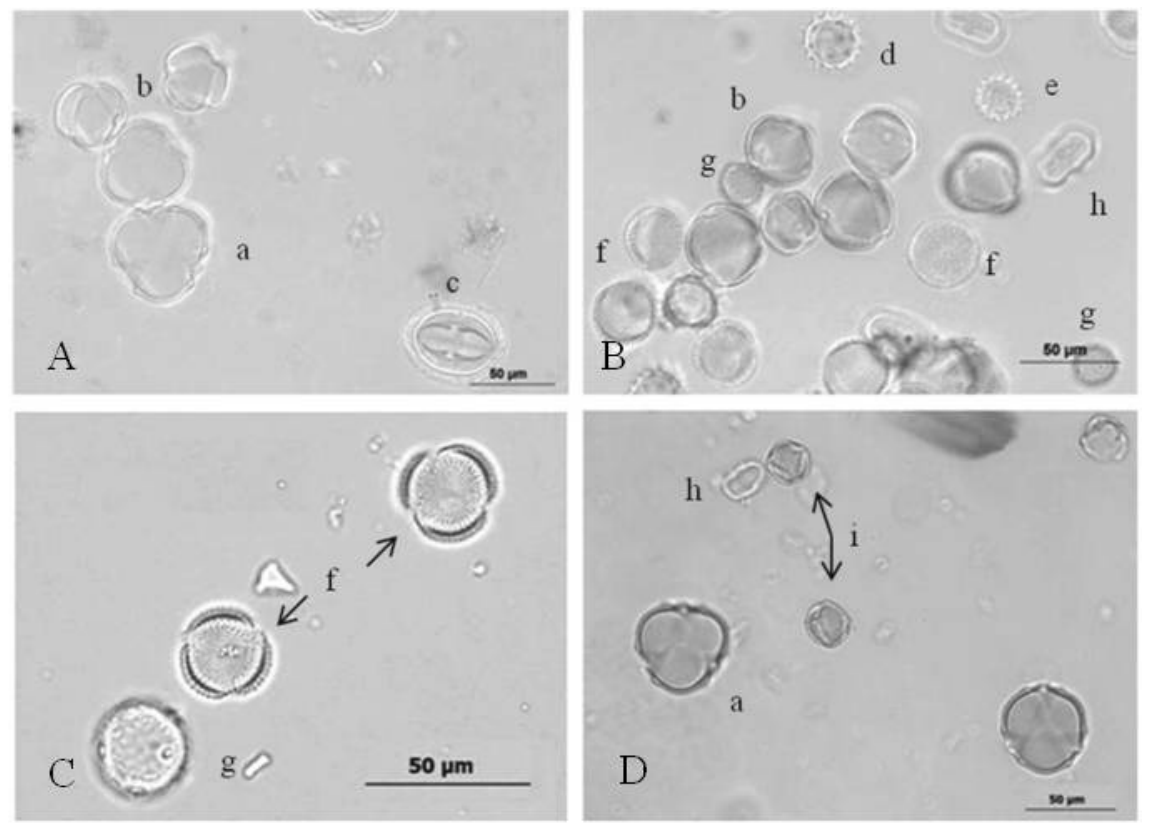

Fig. 3. Micrografías de mieles de la Zona I: Prepuna: A. Muestra 238; B. Muestra 492; C. Muestra 356; D. 220: a. Arquita trichocarpa; b. Prosopis ferox; c. Mutisieae; d. Asteraceae; e. Baccharis sp. f. Brassicaceae; g. Plantago sp.; h. Tipo Papilionoideae; i. Schinus areira.

de polen secundario en cinco y dos muestras respectivamente. Tanto P. ferox como A. trichocarpa son especies nativas de Prepuna, no registrándose en mieles de otros sectores del país, por ello podrían utilizarse como marcadores geográficos.

La familia Brassicaceae, ampliamente distribuida en las zonas templadas del mundo, realizó un importante aporte nectarífero, a través de las especies nativas e introducidas presentes en el área de estudio. Dado la proximidad de los apiarios a zonas de cultivo, es de suponer que el aporte de néctar registrado sea de especies cultivadas como Brassica oleracea L. (con sus diferentes variedades hortícolas) o malezas de cultivos como Rapistrum rugosum (L.) All., Raphanus sativus L. y Lepidium didymum L. Este tipo polínico está presente en el $91 \%$ de las muestras y en cuatro alcanza valores de polen secundario. Las Brassicaceae son un recurso utilizado por abejas en otros ambientes de la provincia (Sánchez, 2013), siempre como polen de menor importancia o en trazas. De igual forma se lo cita en mieles de Entre Ríos (Fagúndez \& Caccavari, 2003, 2006), de San Luis (Costa et al., 1995), Chubut (Forcone \& Tellería, 1998;
2000), alcanzando valores de secundario en el Delta del Paraná (Basilo \& Romero, 1996), en La Pampa (Naab, 1993), en Chubut (Forcone, 2008) y dominante en el Valle de Río Negro (Tellería \& Forcone, 2000; Forcone, 2003) y en sur del distrito del Caldén, provincia fitogográfica del Espinal, en el sur de Buenos Aires y La Pampa (Andrada \& Tellería 2002).

Otro recurso importante hallado en las mieles es Schinus areira (Anacardiaceae), que está presente en el $100 \%$ de las muestras, en tres de ellas como secundario. Esta especie es abundante en toda la Quebrada de Humahuaca, siendo frecuente con hábito arbustivo. El tipo polínico Schinus se encuentra presente en otros ambientes de la provincia, donde también es muy frecuente (Sánchez, 2013), atribuyendo su identidad a Schinus gracilipes Johnst., S. piliferus Johnst. y S. myrtifolius (Griseb.) Cabrera en la provincia fitogeográfica de las Yungas y S. bumelioides I.M. Johnst. y S. fasciculatus (Griseb.) I.M. Johnst. en el Chaco (Muñoz, 2000). En otras regiones del país el tipo polínico Schinus se encuentra citado extensamente en la bibliografía, tanto en la 
provincia fitogeográfica Pampeana (Tellería, 1992; 1996a), del Espinal (Costa et al., 1995; Andrada \& Tellería, 2002; Naab et al., 2008), del Monte y en Patagónica (Forcone, 2008) con clase de frecuencia que varía entre polen secundario y trazas y mieles uniflorales de Schinus patagonicus (Phil.) I.M. Johnst. ex Cabrera, en la provincia Subantártica (Forcone, 2008).

El origen geográfico se define por la presencia en el espectro polínico, con frecuencia de ocurrencia superior al 50\%, de Prosopis ferox, Arquita trichocarpa, Schinus areira, Baccharis sp. y Mutisieae todos estos elementos pertenecen a la vegetación de la Prepuna, confirmando lo encontrado por Sánchez (2013), donde se realizó los análisis de agrupamiento y de correspondencia canónico, observándose que las muestras de esta zona se separan claramente de las demás muestras estudiadas de la provincia de Jujuy. Otros tipos polínicos que aportan a la caracterización geográfica son: el tipo Papilionoideae, que se destaca por su frecuencia de ocurrencia y como recurso nectarífero, de origen indeterminado; las Brassicaceae, que acompañan al hombre en las zonas de cultivos; las Poaceae y Plantago sp., de polinización anemófila y cuya presencia se explica mediante la contaminación, ya sea en la colmena por celdas que almacenan polen o por deposición de la lluvia polínica. Además pueden acompañar tipos polínicos correspondientes a Asteraceae, Solanaceae y Verbenaceae, todas ellas de origen indeterminado.

\section{Conclusiones}

En la Zona I: Prepuna, la flora nativa es importante en la elaboración de las mieles de Apis mellifera, siendo las familias Asteraceae y Fabaceae las más representadas en el espectro polínico.

Debido a la clase de frecuencia y la frecuencia de ocurrencia registrada, se destacan como recursos nectaríferos Prosopis ferox, tipo Papilionoideae, Arquita trichocarpa Brassicaceae y Schinus areira.

La asociación de especies indicadoras de origen geográfico está integrada por: Prosopis ferox, Schinus areira, Arquita trichocarpa, Baccharis sp., Mutisieae acompañados por tipo Papilionoideae, Brassicaceae, Plantago sp., Poaceae, Asteraceae, Solanaceae y Verbenaceae.

\section{Agradecimientos}

Las autoras agradecen a la Lic. Pamela Fierro por su cooperación en la elaboración del mapa, a los apicultores que contribuyeron con sus muestras y a los proyectos SECTER-UNJu que financiaron esta investigación.

\section{Bibliografía}

ANDRADA, A. C. \& M. C. TELLERÍA 2002. Botanical origin of honey from south of Calden district (Argentina). Grana 41: 58-62.

BASILIO, A. M. \& E. J. ROMERO 1996. Contenido polínico en las mieles de la región del Delta del Paraná (Argentina). Darwiniana 34: 113 - 120.

CABRERA, A. L. 1976. Regiones Fitogeográficas Argentinas. Enciclopedia Argentina de Agricultura y Jardinería. II (1): 1 - 85.

CABRERA, M. M. 2006. Caracterización polínica de las mieles de la Provincia de Formosa, Argentina. Rev. Museo Argent. Cienc. Nat., n. s. 8: 135-142.

COSTA, M. C., N. DECOLATTI \& F. GODOY 1995. Análisis polínico de las mieles del norte de la Provincia de San Luis (Argentina). Kurtziana 24: 133-144.

FAGÚNDEZ, G. A. \& M. A. CACCAVARI 2003. Caracterización polínica y organoléptica de algunas mieles monoflorales del centro de la provincia de Entre Ríos, Argentina. Polen 12: 77 - 95.

FAGÚNDEZ, G. A. \& M. A. CACCAVARI 2006. Pollen analysis of honeys from the central zone of the Argentine province of Entre Ríos. Grana 45: 305320 .

FELLER-DEMALSY, M., J. PARENT \& A. STRACHAN 1987. Microscopic analysis of honeys from Alberta, Canadá. J. Apic. Res. 26: 123-132.

FORCONE, A. 2003. Floración y utilización de la flora apícola en el Valle inferior del Río Chubut (Patagonia, Argentina). Bol. Soc. Argent. Bot. 38: 301-310.

FORCONE, A. 2008. Pollen analysis of Honey from Chubut (Argentinean Patagonia). Grana 47: 147-158

FORCONE,A.\& M. C. TELLERÍA 1998. Caracterización palinológica de las mieles del valle inferior del Río Chubut (Argentina). Darwiniana 36: 81-86.

FORCONE, A. \& M. C. TELLERÍA 2000. Caracterización palinológica de las mieles de las mieles del la llanura del Río Senguerr (Chubut, Argentina). Darwiniana 38: 267-271.

GRIMM, E.C. 1992. Tilia and Tilia-graph: pollen spreadsheet and graphics programs. Program and Abstracts, 8th International Palynological Congress, 
Aix-en-Provence (France), September 6-12, 1992, p. 56.

IGLESIAS, M. I., JATO. M. V., M. J. AIRA 1993. Estudio palinológico de mieles de montaña de la provincia de Orense (NW-España). Acta Botánica Malacitana. 18: 119-123.

LOUVEAUX, J., A. MAURIZIO \& G. VORWHOL 1978. Methods of Melisopalinology. Bee World 59: 139-157.

LUSARDI, M., PRADO, D. \& S. GATTUSO 2005. Contenido polínico de las mieles del sur de la Provincia de Santa Fe (Argentina). Bol. Soc. Argent. Bot. 40: 85-90.

MAGYP 2015. Ministerio de Agricultura, Ganadería y Pesca. República Argentina. Alimentos Argentinos. Sector Apícola. Disponible en: http://www. alimentosargentinos.gob.ar [Acceso: 06/10/2015]

MARKGRAF, V. \& H. D'ANTONI 1978. Pollen Flora of Argentina.The University of Arizona Press. Tucson, Arizona, EE.UU.

MUÑOZ, J. D. 2000. Anacardiaceae, en A. HUNZIKER (ed.), Flora Fanerogámica Argentina 65: 1-28. Córdoba: ProFlora.

NAAB, O. 1993. Análisis polínico de mieles de la Provincia de La Pampa, Argentina. Actas de las V Jornadas Pampeanas de Ciencias Naturales 106112 .

NAAB, O. \& M. A. TAMAME, 2007. Flora apícola primaveral en la región del Monte de la Provincia de La Pampa (Argentina). Bol. Soc. Argent. Bot. 42: 251-259.

NAAB, O., M. A. TAMAME \& M. CACCAVARI 2008. Palynological and physicochemical characteristics of the unifloral honey types from central Argentina. Span. J. Agric. Res. 6: 566-576.

OLIVEIRA, P. P. \& F. de A. RIBEIRO DOS SANTOS 2014. Prospecção palinológica em méis da Bahia. Feira de Santana: Print Mídia Editora, Bahía.

PERSANO ODDO, L., PIANA, M. L. \& G. R. D' ALBORE 2007. I Mieli Regionali Ialiani. Caratterizzazione melissopalinologica. Ministero delle Politiche Agricole Alimentari e Forestali. C.R.A. Instituto Sperimentale per la Zoología Agraria, Sezione di Apicultura. Roma. Italia.

PIRE, S. M., ANZÓTEGUI, L. M. \& G. A. CUADRADO 1998. Flora Polínica del Nordeste Argentino, Vol. 1. EUDENE-UNNE. Corrientes, Argentina.

PIRE, S. M., ANZÓTEGUI, L. M. \& G. A. CUADRADO 2002. Flora Polínica del Nordeste Argentino, Vol. 2. EUDENE-UNNE. Corrientes, Argentina.

PIRE, S. M., ANZÓTEGUI, L. M. \& G. A. CUADRADO 2006. Flora Polínica del Nordeste Argentino, Vol. 3. EUDENE-UNNE. Corrientes, Argentina.

RAMÍREZ-ARRIAGA, E., NAVARRO-CALVO, L. A. \& E. DÍAZ-CARBAJAL. 2011. Botanical characterisation of Mexican honeys from a subtropical region (Oaxaca) based on pollen analysis. Grana 50: 40-54.

RICCIARDELLI D'ALBORE, G. 1997. Textbook of Melissopalynology. Apimondia Publishing House. Bucharest.

SÁNCHEZ, A. C. \& L. C. LUPO 2011. Origen Botánico y Geográfico de las Mieles de El Fuerte, Departamento de Santa Bárbara, Provincia de Jujuy. Argentina. Bol. Soc. Argent. Bot. 46: 105-111.

SÁNCHEZ, A. C. 2013. Caracterización botánica y geográfica de las mieles de Apis mellifera L. en la provincia de Jujuy. Tesis doctoral. Doctorado en Ciencias Biológicas. Universidad Nacional de Salta.

SÁNCHEZ, A. C. \& N. D. VIGNALE. 2009. Flora Apícola de la Quebrada de Humahuaca. Arnaldoa 16: $101-108$.

SEIJO, M. C., AIRA, M. J., IGLESIAS, I. \& M. V. JATO 1992. Palynological characterization of honey from La Coruña province (NW Spain). J. Apicul. Res. 31: 149-155.

SEIJO, M. C., JATO, M. V., AIRA, M. J. \& I. IGLESIAS 1994. Caratterizzazione pollinica dei mieli di Lugo (N. O. Spagna). Apicoltura 9:7-18.

SEIJO, M. C., AIRA, M. J., IGLESIAS, I. \& M. V. JATO 1995. Origine botanique et zones de production des miels de Pontevedra (nord-ouest Espagne). Acta Bot. Gallica 142: 235-243.

TELLERÍA, M. C. 1992. Caracterización botánica y geográfica de las mieles de la provincia fitogeográfica Pampeana (Republica Argentina) I: Distrito Oriental. Darwiniana 31: 345-350.

TELLERÍA, M. C. 1995. Plantas de importancia apícola del distrito oriental de la región pampeana (Argentina). Bol. Soc. Argent. Bot. 30: 131-136.

TELLERÍA, M. C. 1996a. Caracterización botánica y geográfica de las mieles de la Provincia Fitogeográfica Pampeana (Republica Argentina) II: Tandilla. Bol. Soc. Argent. Bot. 32: 91 - 94.

TELLERÍA, M. C. 1996b. Caracterización botánica y geográfica de las mieles de la provincia fitogeográfica Pampeana (Republica Argentina) III Noroeste de la Provincia de la Pampa. Darwiniana 34: 245-249.

TELLERÍA, M. C. \& A. FORCONE 2000. El polen de las mieles del Valle de Río Negro, provincia fitogeográfica del Monte. Argentina. Darwiniana 38: 273-277.

VILLANUEVA-GUTIÉRREZ, R., MOGUELORDÓÑEZ, Y. B., ECHAZARRETA-GONZÁLE, C. M. \& G. ARANA-LÓPEZ 2009. Monofloral honeys in the Yucatán Peninsula, Mexico. Grana 48: 214-223.

Recibido el 26 de octubre de 2015, aceptado el 6 de junio de 2016. 